\title{
Fluctuations in the transmission properties of a quantum dot with interface roughness and impurities
}

\author{
S K Kirby, D Z-Y Ting and T C McGill
}

Thomas $\mathrm{J}$ Watson, Sr, Laboratory of Applied Physics, California Institute of Technology, Pasadena, CA 91125, USA

\begin{abstract}
We examine statistical fluctuations in the transmission properties of quantum dots with interface roughness and neutral impurities. For this purpose we employ a supercell model of quantum transport capable of simulating potential variations in three dimensions. We find that sample to sample variations in interface roughness in a quantum dot waveguide can lead to substantial fluctuations in the $n=1$ transmission resonance position, width and maximum, We also find that a strongly attractive impurity near the centre of a quantum dot can reduce these fluctuations. Nevertheless, the presence of more than a single impurity can give rise to a complex resonance structure that varies with impurity configuration.
\end{abstract}

\section{Introduction}

Semiconductor nanostructures exhibiting reduced dimensionality, such as quantum wells, wires and dots, have recently drawn much attention. With characteristic dimensions comparable to the electron de Broglie wavelength, these structures operate in the quantum regime and are sensitive to atomic scale variations in geometry and composition. Defect impurities and interface roughness, for example, can alter transport properties [1-5]. In this paper we study sample to sample transmission resonance fluctuations resulting from variations in interface roughness and neutral impurities in a quantum dot electron waveguide. For this purpose, we have developed a supercell model of quantum transport capable of representing variations in three-dimensional potential.

\section{Method}

Our supercell model is based on the one-band, nearestneighbour, cubic lattice tight-binding Hamiltonian

$$
H=\sum_{n} \varepsilon_{n}|n\rangle\left\langle n\left|+\sum_{\langle n m\rangle} t_{n m}\right| n\right\rangle\langle m|
$$

where the second sum extends over all nearest-neighbour pairs on a cubic lattice of lattice constant $a$. Each of the sites $n$ is associated with two material parameters: a band edge $E_{n}$, and an effective mass, $m_{n}$. In terms of these parameters, the on-site energies $\varepsilon_{n}$ and the hopping matrix elements $t_{n m}$ used in the Hamiltonian are, following
Frensley [6]

$$
\begin{aligned}
\varepsilon_{n} & =E_{n}-\sum_{m} t_{n m} \\
t_{n m} & =\frac{1}{2}\left(t_{n}+t_{m}\right) \\
t_{n} & =-\frac{\hbar^{2}}{2 m_{n} a^{2}} .
\end{aligned}
$$

The sum in the first line above is over all nearestneighbour sites $\boldsymbol{m}$ of site $\boldsymbol{n}$. These definitions are familiar when one considers the special case of a uniform bulk material of band edge $E_{0}$ and effective mass $m$, in which case the Hamiltonian gives rise to the band structure

$$
E(k)=E_{0}-2 t\left(3-\cos k_{x} a-\cos k_{y} a-\cos k_{z} a\right)
$$

where $t=-\hbar^{2} / 2 m a^{2}$.

In order to make quantum transport calculations tractable, we apply a planar supercell method to this Hamiltonian. We model a three-dimensional device structure as a series of monolayer planes along the $z$ direction. Each plane consists of an infinite periodic array of identical rectangular supercells, $n_{x}$ sites in the $x$ direction and $n_{y}$ sites in the $y$ direction, as in figure 1 . The sites for the supercell in a particular plane are chosen to reflect the properties of that plane. For example, if the plane represents a region of bulk material, the sites are identical. To represent a cross-sectional plane of a quantum dot with an impurity we configure the supercell as in figure 1. To calculate quantum transport in this model, we use an efficient, numerically stable method [7-9]. 


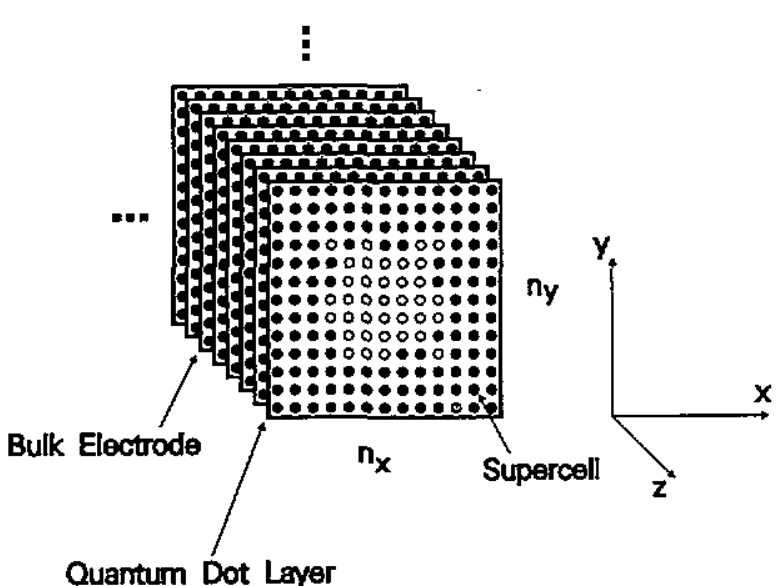

Figure 1. Supercell representation of a quantum dot electron waveguide with rough walls (black) and an impurity (hatched). The supercells repeat in the planes normal to the $z$ direction. In the tight-binding model, an on-site energy corresponds to each site, and a hopping matrix element corresponds to each nearest-neighbour pair of sites.

\section{Results and discussion}

We first study fluctuations in a set of quantum dots with interface (micro-)roughness. Each structure consists of a $2.5 \mathrm{~nm} \times 2.5 \mathrm{~nm} \times 3.5 \mathrm{~nm}$ cavity of well-type material surrounded by barrier material. The barrier is $10 \mathrm{~nm}$ thick at each end, and the device is sandwiched between two electrodes along the $z$ direction. Between the well and the barrier is a $0.5 \mathrm{~nm}$ thick shell of interface roughness consisting of an uncorrelated random distribution of $50 \%$ well-type sites and $50 \%$ barrier-type sites (cf figure 1). Transmission coefficient curves for 10 such devices with different configurations of interface roughness are plotted in the top panel of figure 2 , along with curves for two ideal, smooth-walled quantum dots $2.5 \mathrm{~nm} \times$ $2.5 \mathrm{~nm} \times 3.5 \mathrm{~nm}$ and $3.5 \mathrm{~nm} \times 3.5 \mathrm{~nm} \times 4.5 \mathrm{~nm}$. We see that the $n=1$ resonance position of the rough-walled samples varies over a range comparable with the resonance width. Resonance widths and maximum transmission coefficients for the 10 samples are plotted in the bottom panel, normalized so that average values are 1 . We see that there is roughly a $10-20 \%$ variation in the resonance width and roughly a $5 \%$ variation in the maximum transmission. Also plotted for scale are the resonance widths and maxima for the two ideal structures. Both the widths and maxima for the 10 samples show substantial fluctuation on this scale.

These large fluctuations can be understood via an analysis of the electron wavefunction at the resonance. We first calculate the total electron probability density in the quantum dot structure, including all sites in the supercells containing barrier material. We then calculate the total electron probability density in the $0.5 \mathrm{~nm}$ shell of interface roughness and express this as a percentage of the total. At the $n=1$ resonance in a dot with interface roughness, about $27.2 \%$ lies in the shell containing the roughness. Thus electrons sample the roughness substan-
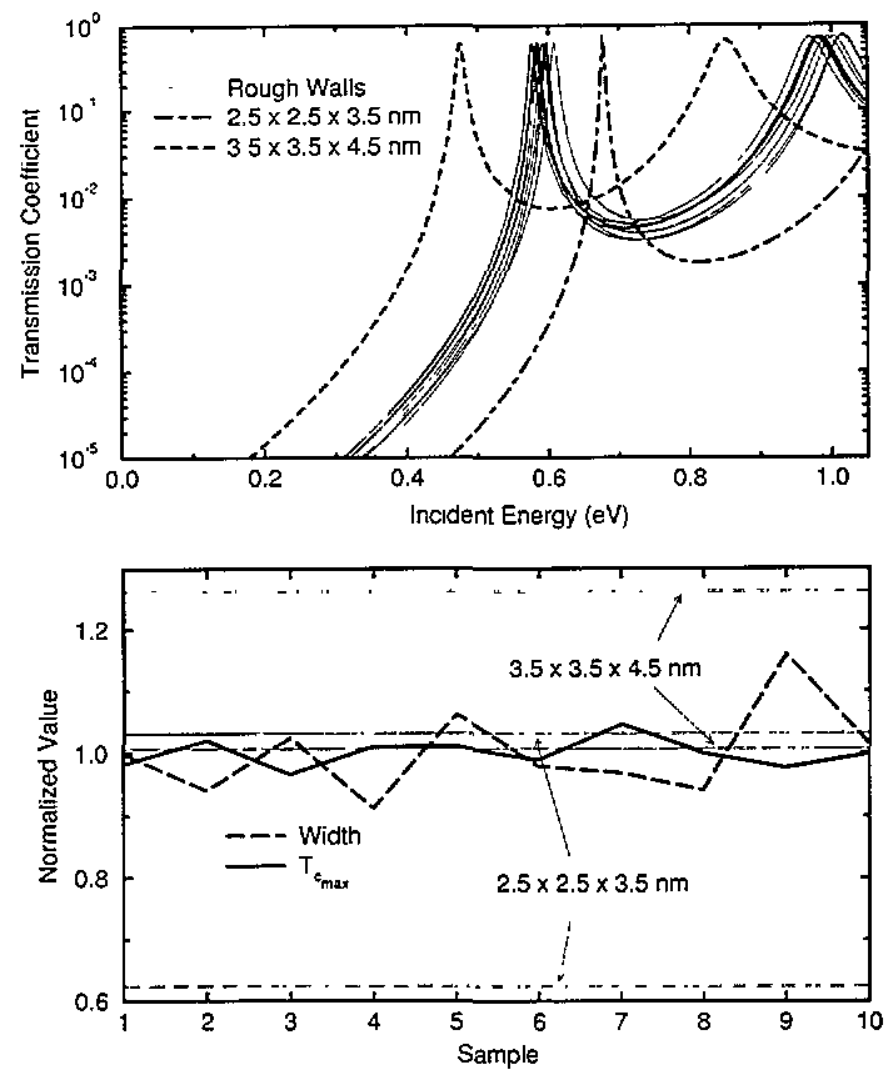

Figure 2. Top panel: transmission coefficient curves for quantum dots with 10 different rough-walled configurations, as described in the text, and for two ideal, smooth-walled dots. Bottom panel: $n=1$ resonance widths and maxima for the 10 samples and for the ideal dots: $a=0.5 \mathrm{~nm}, 13 \times 13$ supercell, $E_{\text {electrode }}=-1 \mathrm{eV}$, $m_{\text {electrode }}=0.1 m_{0}, E_{\text {walls }}=1.05 \mathrm{eV}, m_{\text {walls }}=0.1248 m_{0}$, $E_{\text {dot }}=0 \mathrm{eV}, m_{\text {cot }}=0.0673 m_{0}$. Plane waves are incident along the $z$ direction.

tially, and variations in the roughness can be expected to have a significant impact. This suggests that, if the resonance mode could be altered so as to draw the resonant wave function probability density away from the roughness, fluctuations might be reduced.

We place an attractive impurity at the centre of the quantum dot as a means of drawing the electron probability density in toward the impurity site to reduce the interaction with the rough interface. To demonstrate, we calculate transmission coefficient curves for the same set of rough-walled dots, but with a strongly attractive impurity in the centre, represented by a single site whose on-site energy is $\Delta U$ below that of the surrounding sites. The hopping matrix element to the site, $t$, is the same as that in the surrounding material. In the top panel of figure 3 we plot the results using $\Delta U / t=-4.9$. For these parameters, only $1.4 \%$ of the $n=1$ resonant wavefunction probability density lies in the shell of roughness.

A glance at figure 3 reveals that the $n=1$ resonance fluctuates over a much narrower energy range than without the impurity. These resonances, at considerably lower energy and much sharper than the $n=1$ resonances in figure 2 , have more of the character of an impurity resonance than a cavity resonance of the quantum dot. 

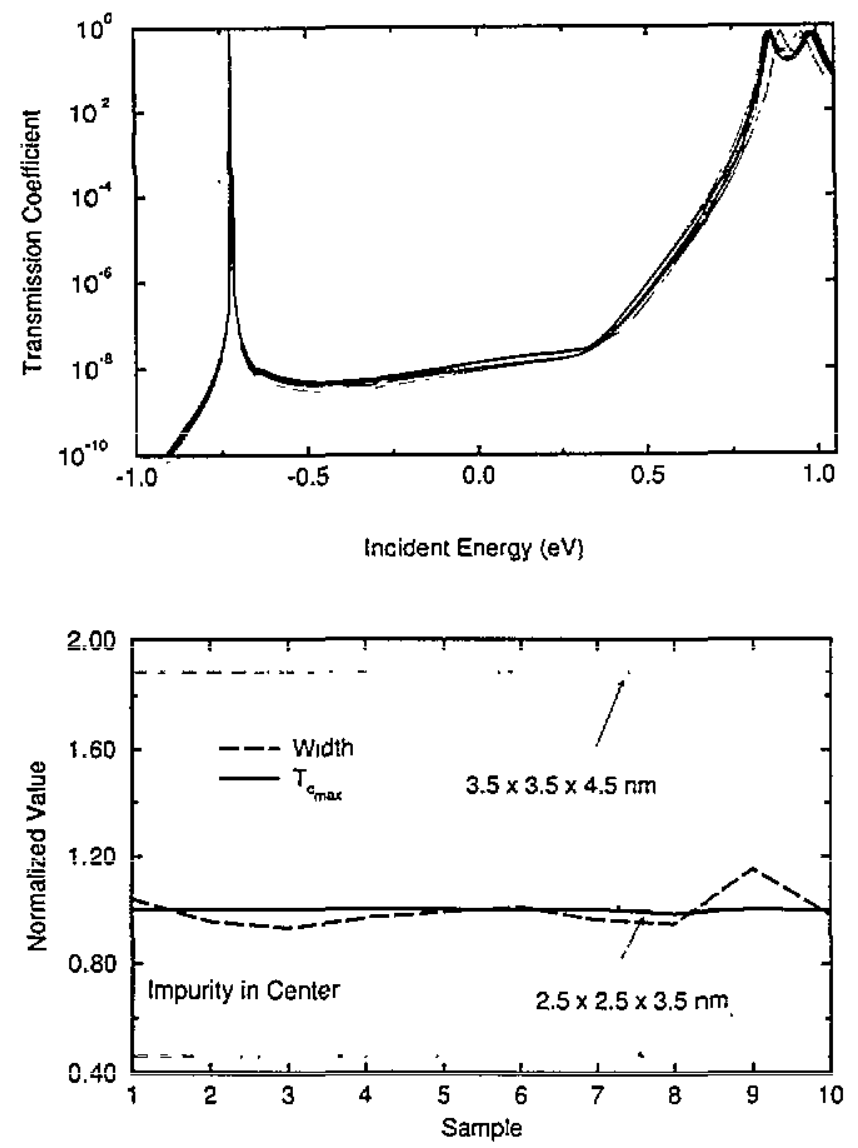

Figure 3. Top panel: transmission coefficient curves for the 10 rough-walled quantum dots of figure 2 , each with a strongly attractive $(\Delta U / t \approx-4.9)$ impurity in the centre. Bottom panel: $n=1$ resonance widths and maxima for the 10 samples and for ideal dots with an impurity in the centre: $a=0.5 \mathrm{~nm}, 13 \times 13$ supercell, $E_{\text {electrode }}=-1 \mathrm{eV}$, $m_{\text {electrode }}=0.1 m_{0}, E_{\text {walls }}=1.05 \mathrm{eV}, m_{\text {walls }}=0.1248 m_{0}$, $E_{\text {dot }}=0 \mathrm{eV}, m_{\text {dot }}=0.0673 m_{0}$. Plane waves are incident along the $z$ direction.

This contributes to the reduction in fluctuations. The resonance width and maximum transmission also fluctuate less, as shown in the bottom panel. Here the widths and maxima of the $n=1$ resonances of the 10 samples are plotted, normalized so that their average values are 1 . Also shown for reference are the widths and maxima of the $n=1$ resonances of the two ideal dots of figure 2, but with an impurity of strength $\Delta U / t=-4.9$ at the centre. It is evident that the attractive impurity reduces fluctuations.

If more than a single impurity is present, however, fluctuations still pose a problem: different impurity configurations at the same concentration can lead to different transmission spectra. To demonstrate this, we plot, in figure 4 , transmission coefficient curves for the rough-walled dot of sample 1 in figure 2 with two different configurations of impurities in the cavity. Each configuration consists of 11 impurity sites placed at random among the 175 sites in the quantum dot. The high concentration of impurities produces a complex resonance structure, whose peak positions, widths and maxima depend on the configuration.

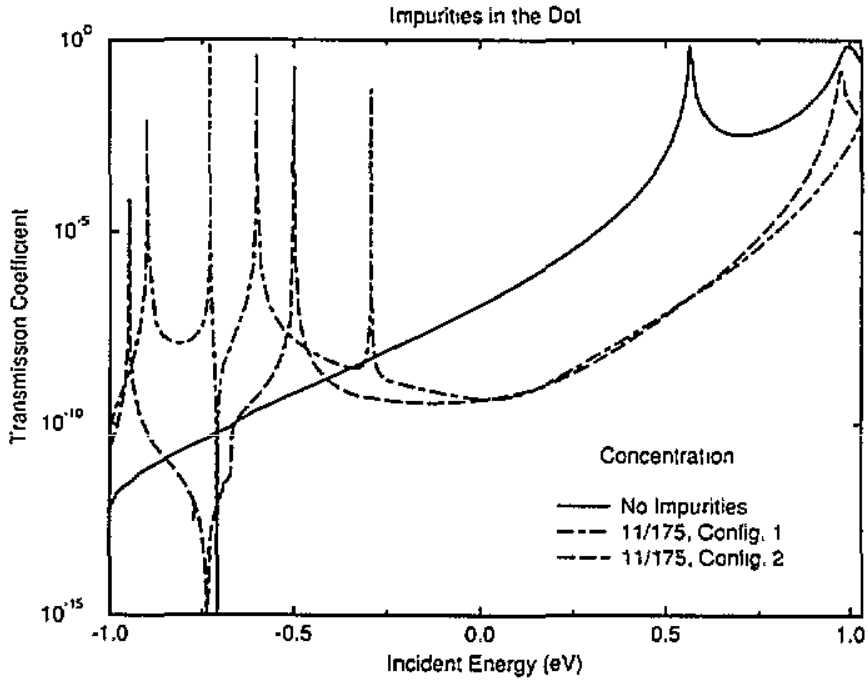

Figure 4. Transmission coefficient curves for a rough-walled quantum dot with a concentration of $0.063 / a^{3}$ strongly attractive $(\Delta U / t \approx-4.9)$ impurities in the cavity (11 impurity sites were chosen at random out of the 175 sites within the cavity.) Also shown is the transmission coefficient curve for the rough-wailed dot without impurities: $a=0.5 \mathrm{~nm}, 13 \times 13$ supercell, $E_{\text {electrode }}=-1 \mathrm{eV}, m_{\text {electrode }}=0.1 m_{0}, E_{\text {walls }}=1.05 \mathrm{eV}$, $m_{\text {walls }}=0.1248 m_{0}, E_{\text {dot }}=0 \mathrm{eV}, m_{\text {dot }}=0.0673 m_{0}$. Plane waves are incident along the $z$ direction.

\section{Conclusion}

We have seen that sample to sample variations in interface roughness in a quantum dot waveguide can lead to substantial fluctuations in the $n=1$ transmission resonance position, width and maximum. These fluctuations in the transmission resonance could, in turn, lead to fluctuations in the current-voltage characteristicsthe peak current magnitude, bias condition and the peak to valley current ratio could vary from sample to sample. We have also seen that a strongly attractive impurity near the centre of the dot can reduce these fluctuations. Nonetheless, the presence of more than a single impurity in the dot can lead to a complex, impurity-configurationdependent resonance structure, especially at high concentrations. If quantum structures are to form the basis of mass-produced electronic devices, the issue of fluctuations must be tackled. Indeed there is much hope, though many challenges remain.

\section{Acknowledgments}

The authors would like to thank Dr Eric F Van de Velde for helpful discussion. S K Kirby would like to thank the Office of Naval Research (ONR) for graduate fellowship support during this work. This work was also supported by the Office of Naval Research under grant no N00014-89-J-1141. 


\section{References}

[1] Mitin V V 1990 Superlatt. Microstruct. 8413

[2] Mceuen P L, Alphenaar B W and Wheeler R G 1990 Sturf. Sci. 229312

[3] Eugster C C, del Alamo J A, Melloch M R and Rooks M J 1992 Phys. Rev. B 4610146

[4] Bagwell P F 1990 Phys. Rev. B 41 10354;

Kumar A and Bagwell P F 1991 Phys. Rev. B 43 9012;
Bagwell P F, Orlando T P and Kumar A 1991 Resonant Tunnelling in Semiconductors ed L L Chang et al (New York: Plenum) p 417

[5] Nixon J A, Davies J H and Baranger H U 1991 Phys. Rev. B 4312638

[6] Frensley W R 1990 Rev. Mod. Phys. 62745

[7] Lent C S and Kirkner D J 1990 J. Appl. Phys. 676353

[8] Frensley $W R$ private communication

[9] Ting D Z-Y, Yu E T and McGill T C 1992 Phys. Rev. B 453583 